\title{
Experimental study of friction in aluminium bolted joints
}

\author{
D. Croccolo $0^{1, a}, M$. De A gostinis ${ }^{1}, N$. Vincenzi ${ }^{1}$ \\ ${ }^{1}$ DIEM Faculty of Engineering, University of Bologna, V .le Risorgimento 2, 40136 B ologna, Italy
}

\begin{abstract}
This study aims at developing an experimental tool useful to define accurately the friction coefficients in bolted joints and, therefore, at relating precisely the tightening torque to the bolt preloading force in some special components used in front motorbike suspensions. The components under investigation are some clamped joints made of aluminium alloy. The preloading force is achieved by applying a torque wrench to the bolt head. Some specific specimens have been appropriately designed and realized in order to study the tribological aspects of the tightening phase. Experimental tests have been performed by applying the Design of Experiment (DOE) method in order to obtain a mathematical model for the friction coefficients. Three replicas of a full factorial DOE at two levels for each variable have been carried out. The levels include cast versus forged aluminium alloy, anodized versus spray-painted surface, lubricated versus unlubricated screw, and first tightening (fresh unspoiled surfaces) versus sixth tightening (spoiled surfaces). The study considers M 8x1.25 8.8 galvanized screws.
\end{abstract}

\section{Introduction}

Threaded fasteners are widely used in mechanical and structural applications because of their easiness to assembly and disassembly for maintenance and repair purposes. B oth the level and the stability of the clamp loads, which are created by the tightening process, will govern the safety and reliability of bolted joints. In most of production applications, the fastener tension (preloading force) is achieved by using a torque wrench applied to the head or to the nut. The tribological aspects of the tightening phase are critical to define the actual torque-tension relationship [1, 2]. The tightening torque is mostly consumed in overcoming two friction components: the underhead (or bearing) friction due to the sliding of the fastener head on the flanges and the thread friction between the male and female thread. The residual torque component produces the fastener tension by generating the joint clamping force. Inaccuracies in determining the friction components may lead to an overestimation or underestimation of the bolted joint performances. The torque-preloading relationship is often simplified by using a constant $\mathrm{K}$ (Eq.1), known as torque coefficient (or nut factor): some A uthors and Standards $[3,4,5]$ provide an approximate value of 0.2 for the nut factor, but cautions against using it for critical joints shall be considered. Bickford [6] provides some mean values of the torque coefficient for various combinations of joint materials and surface conditions. However, the scatter in the torque coefficient is too great to provide a unique and reliable value, particularly in critical joints.

\footnotetext{
a e-mail : dario.croccolo@ unibo.it
} 


$$
T=K \cdot F_{V} \cdot d
$$

$T[\mathrm{Nmm}]$ is the input tightening torque applied to the fastener head or nut, $F_{V}[\mathrm{~N}]$ is the preloading force and $d[\mathrm{~mm}]$ is the nominal thread diameter.

Motosh [7] and VDI2230 [8] provided a more accurate $T-F_{V}$ relationship, reported in Eq.2, as addition of three different contributions: the pitch $T_{p}$, the thread $T_{t}$ and the underhead $T_{u}$ torque.

$$
\begin{aligned}
& T=T_{p}\left(F_{V}, p\right)+T_{t}\left(F_{V}, \mu_{t}, d_{2}\right)+T_{u}\left(F_{V}, \mu_{u}, d_{u}\right) \\
& T=F_{V} \cdot\left[0.16 \cdot p+0.58 \cdot \mu_{t} \cdot d_{2}+0.5 \cdot \mu_{u} \cdot d_{u}\right]
\end{aligned}
$$

$p[\mathrm{~mm}]$ is the thread pitch, $\mu_{t}$ is the friction coefficient between male and female threads, $d_{2}[\mathrm{~mm}]$ is the mean thread diameter $\left(d_{2}=d-0,6495^{\circ} p\right), \mu_{u}$ is the underhead friction coefficient and $d_{u}[\mathrm{~mm}]$ is the underhead mean diameter. The pitch torque component $T_{p}[\mathrm{Nmm}]$ creates the fastener tension and the joint clamp load $F_{V}$. By considering a M8x1.25 bolt $\left(d_{2}=7.19 \mathrm{~mm}, d_{u}=11 \mathrm{~mm}\right)$ and a mean value for friction coefficients $\mu_{t}$ and $\mu_{u}$ equal to 0.15 , it is easy to calculate that $T_{p} \approx 0.12 T, T_{\tau} \approx 0.38 T$ and $T_{u} \approx 0.50 T$ : the $88 \%$ of the total input torque $T$ is consumed in overcoming friction (only $12 \%$ is converted in preloading force).

This study aims at developing an experimental tool and procedure useful to define accurately the friction coefficients in bolted joints and, therefore, at relating precisely the input bolt torque $T$ to the bolt preloading force $F_{V}$. The components under investigation (Fig.1) are some clamped joints made of aluminium alloy used in front motorbike suspensions to connect the steering plates (forks) to the legs and the legs to the wheel pin: the tensile state of these components (maximum stress and coupling pressure) has been deeply studied by the Authors via Finite Elements Analysis (FEA) in a previous work [9], and it has been related to the preloading force. By leveraging the accurate knowledge of the friction coefficients, it is possible to extend the results presented in [9] using, as input parameter for calculation, the more appropriate tightening torque $T$ (actually controlled during the clamping phase) instead of the preloading force $F_{V}$.

The aluminium alloy under investigation is realised by a casting or a forging process, afterwards anodized or spray-painted in surface.

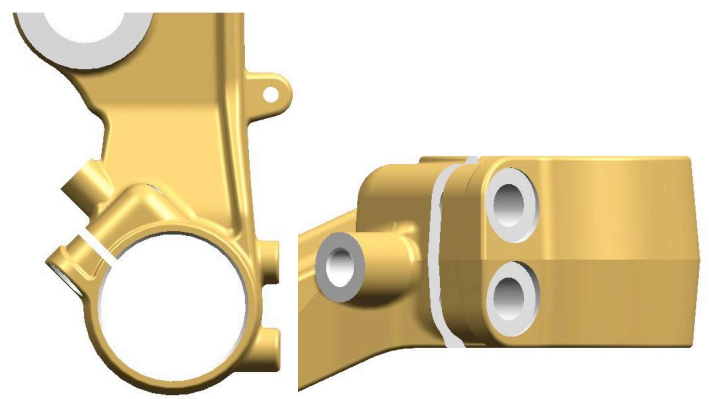

(a)
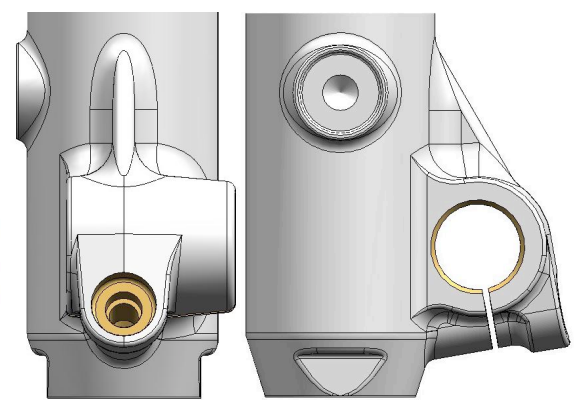

(b)

Fig. 1. Example of bolted joints in front motorbike suspensions: (a) steering plate-leg, (b) leg-wheel pin

\section{Methodology and Results}

In order to evaluate accurately the friction coefficients and to gather their effective mathematical expression, the Design of Experiment method has been applied. In detail, after some screening analyses, a full factorial plane, characterized by 4 variables with 2 levels each, has been designed. Three replicas have been carried out, in order to reduce the influence of noise (experimental error) and any non-investigated factors: a total of $3 \times 2^{4}=48$ experimental tests have been carried out. In Tab. 1 the DOE parameters are summarized. 
Table 1. The Design of Experiment (DOE) parameters: variables and levels.

\begin{tabular}{|c|c|c|}
\hline Variable & Low Level (0) & High level (1) \\
\hline A. Lubrication & Unlubricated & Lubricated \\
\hline B. Process & Cast & Forged \\
\hline C. Surface finishing & Spray-painted & Anodized \\
\hline D. Tightening & First tightening (unspoiled surfaces) & Sixth tightening (spoiled surfaces) \\
\hline
\end{tabular}

According to VDI2230 [8] and DIN946 [10], Eq.2 has been rewritten into Eq.3, considering $\mu_{t}$ and $\mu_{u}$ equal to the same value $\mu_{m}$ in order to describe the overall friction behaviour of the bolt/nut assemblies.

$$
\begin{aligned}
& T=F_{V} \cdot\left[0.16 \cdot p+0.58 \cdot \mu_{m} \cdot d_{2}+0.5 \cdot \mu_{m} \cdot d_{u}\right] \\
& \mu_{m}=\frac{T / F_{V}-0.16 \cdot p}{0.58 \cdot d_{2}+0.5 \cdot d_{u}}
\end{aligned}
$$

Each test planned by the DOE provides a value of the overall friction coefficient $\mu_{m}$ (Eq.3): in order to calculate $\mu_{m}$ some specific specimens, reported in Fig.2, have been appropriately designed and realized with the same process (cast or forged) and surface finishing (spray-painted or anodized) of the actual components (Fig.1). The study considers M8x1.25, SAE Standard 8.8, galvanized screws $\left(d_{2}=7.19 \mathrm{~mm} ; d_{u}=11 \mathrm{~mm}\right)$.
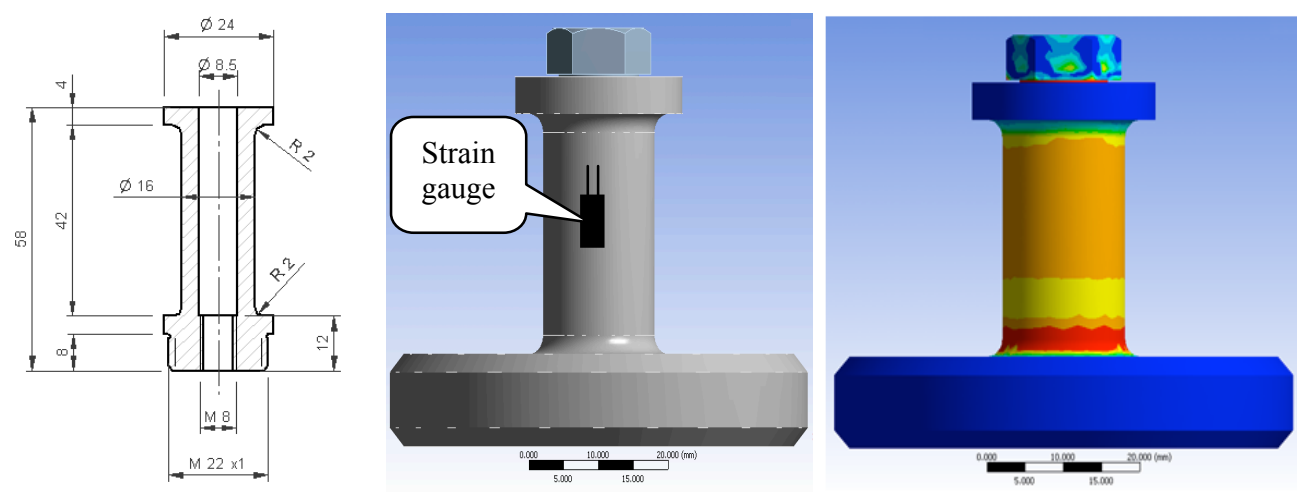

Fig.2 The specimen used for the friction coefficient $\mu_{m}$ definition

The total torque $T$ is given by a calibrated torque wrench whereas the preloading force $F_{V}$ has been evaluated by means of a strain gauge, located on the external surface of the specimen, which is able to provide the axial compression strain $\varepsilon_{C}$. The compression force $F_{C}$ (Eq.4) acting on the specimen is equal, in magnitude, to the preloading force acting on the screw, since the system works like series of mechanical stiffness during the tightening phase.

$$
\left|F_{C}\right|=F_{V}=\left|\varepsilon_{C}\right| \cdot E \cdot A_{e q}
$$

$E[\mathrm{MPa}]$ is the Young's modulus of the aluminium specimen $(69.000 \mathrm{MPa})$ and $A_{e q}\left[\mathrm{~mm}^{2}\right]$ is the cylindrical equivalent cross section of the compressed region, when the external diameter of the bush $\left(D_{A}=16 \mathrm{~mm}\right)$ exceeds the bolt head diameter $\left(d_{w}=13 \mathrm{~mm}\right)$ as indicated in the sketch of Fig. 3. 


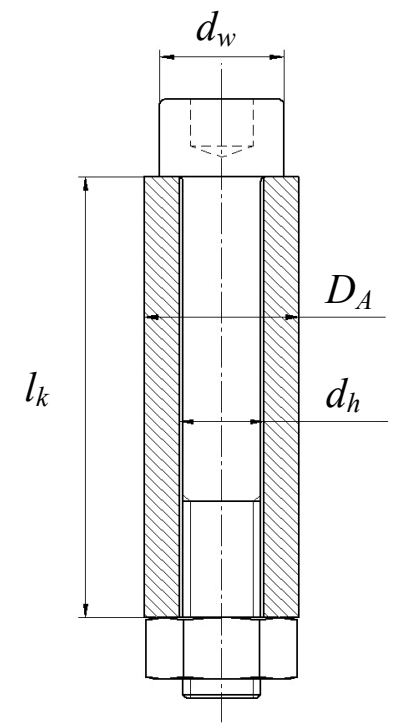

Fig.3 Sketch of a bolt-bush joint

In order to evaluate $A_{e q}$ some formulae, as functions of different geometries (internal diameter of the specimen $d_{h}=8.5 \mathrm{~mm}$, length of the specimen $l_{k}=4+42=46 \mathrm{~mm}$ ), are proposed in [11]: the equivalent cross section (Eq.5) results equal to $143.6 \mathrm{~mm}^{2}$.

$$
A_{e q}=\frac{\pi}{4} \cdot\left(d_{w}{ }^{2}-d_{h}{ }^{2}\right)+\frac{\pi}{8} \cdot d_{w} \cdot\left(D_{A}-d_{w}\right) \cdot\left[\left(\sqrt[3]{\frac{l_{k} \cdot d_{w}}{D_{A}{ }^{2}}}+1\right)^{2}-1\right]=143.6 \mathrm{~mm}^{2}
$$

Since the specimen has been designed to be very similar to a thin bush, the equivalent cross section $A_{e q}$ can be considered equal to the actual cross area $A$ (Eq.6): the difference is equal to $0.5 \%$.

$$
A=\frac{\pi}{4} \cdot\left(D_{A}^{2}-d_{h}^{2}\right)=144.3 m m^{2} \approx A_{e q}
$$

A Finite Element Analysis (FEA), reported in Fig.2, has demonstrated that the whole cross section (circular and hollow), in correspondence of the region where the strain gauge is located, has the same strain and, therefore, the same stress: the proposed tool (specimen and strain gauge) is able to provide an accurate estimation of the preloading force (Eq.4).
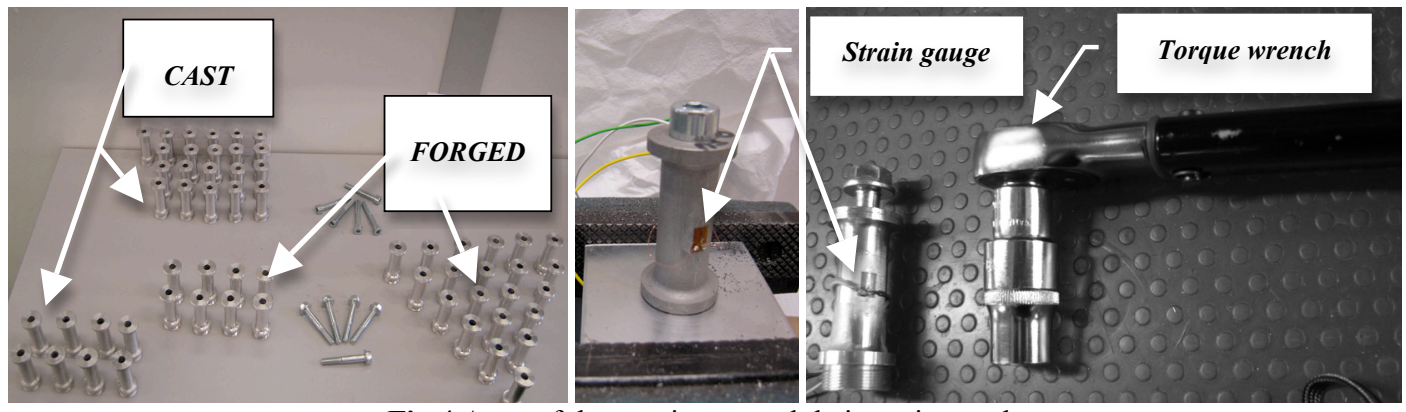

Fig.4 A set of the specimens and their testing tools 
According to [12], experimental tests have been randomized: a total torque $T$ equal to $15 \mathrm{Nm}$ has been applied to each specimen. The preloading force $F_{V}$ has been evaluated, as reported in Fig. 4 and both the overall friction coefficient $\mu_{m}$ (Eq.3) and the torque coefficient $K$ (Eq.1) have been calculated, as functions of the DOE variables and levels (Tab.1). The DOE plan and results (48 tests) are reported in Tab.2.

Table 2. DOE plan and results provided by experimental tests $(T=15 \mathrm{Nm})$

\begin{tabular}{||c|c|c|c|c|c|c|c||c|c|c|c|c|c|c|c||}
\hline \hline ID & $\begin{array}{c}\text { A. } \\
\text { Lubrication }\end{array}$ & $\begin{array}{c}\text { B. } \\
\text { Process }\end{array}$ & $\begin{array}{c}\text { C. } \\
\text { Surface } \\
\text { finishing }\end{array}$ & $\begin{array}{c}\text { D. } \\
\text { Tightening }\end{array}$ & $\begin{array}{c}\boldsymbol{F}_{\boldsymbol{V}} \\
{[\mathbf{N}]}\end{array}$ & $\boldsymbol{\mu}_{\boldsymbol{m}}$ & $\boldsymbol{K}$ & $\mathbf{I D}$ & $\mathbf{A .}$ & $\mathbf{B .}$ & $\mathbf{C}$. & $\mathbf{D .}$ & $\begin{array}{c}\boldsymbol{F}_{\boldsymbol{V}} \\
{[\mathbf{N}]}\end{array}$ & $\boldsymbol{\mu}_{\boldsymbol{m}}$ & $\boldsymbol{K}$ \\
\hline 1 & 1 & 0 & 1 & 0 & 10102 & 0.13 & 0.19 & 13 & 1 & 1 & 1 & 1 & 10102 & 0.13 & 0.19 \\
\hline 2 & 1 & 1 & 1 & 0 & 9092 & 0.15 & 0.21 & 16 & 0 & 1 & 1 & 1 & 3608 & 0.41 & 0.52 \\
\hline 3 & 0 & 1 & 1 & 0 & 6783 & 0.21 & 0.28 & 15 & 1 & 0 & 0 & 1 & 15298 & 0.08 & 0.12 \\
\hline 4 & 0 & 0 & 1 & 0 & 7504 & 0.19 & 0.25 & 10 & 1 & 1 & 0 & 1 & 18040 & 0.07 & 0.10 \\
\hline 5 & 0 & 1 & 0 & 0 & 10391 & 0.13 & 0.18 & 14 & 1 & 0 & 1 & 1 & 15153 & 0.08 & 0.12 \\
\hline 6 & 1 & 0 & 0 & 0 & 17751 & 0.07 & 0.11 & 11 & 0 & 0 & 1 & 1 & 4041 & 0.36 & 0.46 \\
\hline 7 & 0 & 0 & 0 & 0 & 14865 & 0.08 & 0.13 & 12 & 0 & 0 & 0 & 1 & 10102 & 0.13 & 0.19 \\
\hline 8 & 1 & 1 & 0 & 0 & 17895 & 0.07 & 0.10 & 9 & 0 & 1 & 0 & 1 & 9814 & 0.14 & 0.19 \\
\hline 8 & 1 & 1 & 0 & 1 & 16019 & 0.08 & 0.12 & 17 & 1 & 1 & 1 & 0 & 9525 & 0.14 & 0.20 \\
\hline 4 & 0 & 0 & 1 & 1 & 5051 & 0.29 & 0.37 & 18 & 0 & 0 & 1 & 0 & 6783 & 0.21 & 0.28 \\
\hline 2 & 1 & 1 & 1 & 1 & 10679 & 0.12 & 0.18 & 19 & 0 & 1 & 0 & 0 & 11978 & 0.11 & 0.16 \\
\hline 5 & 0 & 1 & 0 & 1 & 7793 & 0.18 & 0.24 & 20 & 1 & 1 & 0 & 0 & 16885 & 0.07 & 0.11 \\
\hline 7 & 0 & 0 & 0 & 1 & 12267 & 0.11 & 0.15 & 21 & 0 & 0 & 0 & 0 & 11257 & 0.12 & 0.17 \\
\hline 3 & 0 & 1 & 1 & 1 & 4185 & 0.35 & 0.45 & 22 & 0 & 1 & 1 & 0 & 4762 & 0.31 & 0.39 \\
\hline 6 & 1 & 0 & 0 & 1 & 16019 & 0.08 & 0.12 & 23 & 1 & 0 & 1 & 0 & 9669 & 0.14 & 0.19 \\
\hline 1 & 1 & 0 & 1 & 1 & 10824 & 0.13 & 0.18 & 24 & 1 & 0 & 0 & 0 & 9381 & 0.14 & 0.20 \\
\hline 9 & 0 & 1 & 0 & 0 & 14432 & 0.09 & 0.13 & 18 & 0 & 0 & 1 & 1 & 4330 & 0.34 & 0.43 \\
\hline 10 & 1 & 1 & 0 & 0 & 21648 & 0.05 & 0.09 & 21 & 0 & 0 & 0 & 1 & 9381 & 0.14 & 0.20 \\
\hline 11 & 0 & 0 & 1 & 0 & 5484 & 0.26 & 0.34 & 22 & 0 & 1 & 1 & 1 & 2165 & 0.70 & 0.87 \\
\hline 12 & 0 & 0 & 0 & 0 & 12989 & 0.10 & 0.14 & 23 & 1 & 0 & 1 & 1 & 8659 & 0.16 & 0.22 \\
\hline 13 & 1 & 1 & 1 & 0 & 8515 & 0.16 & 0.22 & 19 & 0 & 1 & 0 & 1 & 8659 & 0.16 & 0.22 \\
\hline 14 & 1 & 0 & 1 & 0 & 15009 & 0.08 & 0.12 & 17 & 1 & 1 & 1 & 1 & 9525 & 0.14 & 0.20 \\
\hline 15 & 1 & 0 & 0 & 0 & 16885 & 0.07 & 0.11 & 24 & 1 & 0 & 0 & 1 & 9236 & 0.15 & 0.20 \\
\hline 16 & 0 & 1 & 1 & 0 & 6206 & 0.23 & 0.30 & 20 & 1 & 1 & 0 & 1 & 15442 & 0.08 & 0.12 \\
\hline \hline
\end{tabular}

Table 3. Example of ANOVA Table results for ${ }_{m}$

\begin{tabular}{|c|c|c|c|c|}
\hline Effect (interaction) & Sum of Square & DoF & Mean Square & $\boldsymbol{F}_{\boldsymbol{~}}$ \\
\hline A - Lubrication & 0.1610 & 1 & 0.1610 & $\mathbf{5 3 . 3 1}$ \\
\hline B - Process & 0.0085 & 1 & 0.0085 & 2.83 \\
\hline C - Surface finishing & 0.1776 & 1 & 0.1776 & $\mathbf{5 8 . 8 1}$ \\
\hline D - Number of tightening & 0.0352 & 1 & 0.0352 & $\mathbf{1 1 . 6 6}$ \\
\hline AB & 0.0114 & 1 & 0.0114 & 3.78 \\
\hline AC & 0.0690 & 1 & 0.0690 & $\mathbf{2 2 . 8 5}$ \\
\hline AD & 0.0320 & 1 & 0.0320 & 10.61 \\
\hline BC & 0.0108 & 1 & 0.0108 & 3.58 \\
\hline BD & 0.0030 & 1 & 0.0030 & 1.00 \\
\hline CD & 0.0102 & 1 & 0.0102 & 3.38 \\
\hline ABC & 0.0004 & 1 & 0.0004 & 0.14 \\
\hline ABD & 0.0056 & 1 & 0.0056 & 1.87 \\
\hline ACD & 0.0176 & 1 & 0.0176 & 5.84 \\
\hline BCD & 0.0010 & 1 & 0.0010 & 0.33 \\
\hline Error & 0.0997 & 33 & 0.0030 & 1.00 \\
\hline
\end{tabular}

Once the whole 48 values of $\mu_{m}$ and $K$ have been calculated, their results were studied by applying the ANalysis Of VAriance (ANOVA) $[12,13]$. This technique is based on a statistical approach and allows evaluating the effect of each variable and of their interaction, in order to identify those variables (or interactions) that are actually significant in changing the friction coefficients value. By means of the software Stagraphics ${ }^{\circledR}$ Plus, Release 5.1, the ANOVA table has been generated (an 
example is reported in Tab.3) and the Fisher's test (F-Test) has been executed. $F_{0}$ value is determined subdividing the Mean Square of the single effect (or interaction) with the Mean Square of the Error [14]. The error probability $\alpha$ has been set equal to $0.10(10 \%)$ and Fisher's value $F_{v 1 ; v 2 ; \alpha}$ has been obtained from Fisher's table $[12,13]\left(v_{l}\right.$ are the Degrees of Freedom of the source of variation and $v_{2}$ of the source of Error). The variables (or interactions) with $F_{0}$ higher than the corresponding $F_{v 1 ; v 2 ; \alpha}\left(F_{1 ; 17 ; 10 \%}=2.87\right)$ are significant in the analysis (grey cells in Tab.3), whereas the other could be ignored in the response model (white cells in Tab.3), with a confidential level of $(1-\alpha)=90 \%$. ANOVA and F-Test provide Eq. (7) and Eq. (8) in which the Regression Coefficients and, therefore, the mathematical models for $\mu_{m}$ and $K$ are reported as functions of the significant investigated variables (or interactions).

$$
\begin{aligned}
& \mu_{\mathrm{m}}=0.108-0.023 \cdot A+0.103 \cdot C+0.010 \cdot D-0.007 \cdot A \cdot B-0.063 \cdot A \cdot C+ \\
& +0.017 \cdot A \cdot D+0.053 \cdot B \cdot C+0.117 \cdot C \cdot D-0.153 \cdot A \cdot C \cdot D \\
& K=0.157-0.028 \cdot A+0.123 \cdot C+0.013 \cdot D-0.008 \cdot A \cdot B-0.075 \cdot A \cdot C+ \\
& +0.015 \cdot A \cdot D+0.065 \cdot B \cdot C+0.138 \cdot C \cdot D-0.180 \cdot A \cdot C \cdot D
\end{aligned}
$$

Surface finishing (specimen spray-painted or anodized), lubrication (specimen lubricated or unlubricated), the interaction between surface finishing and lubrication, and finally the number of tightening are the most significant parameters (highest values of $F_{0}$ are highlighted by the bold style in Tab.3). In the bars diagrams of Fig. 5 and Fig. 6 some results are compared depending on the aforementioned parameters in case of forged specimens.

It is possible to highlight that the spray-painted specimens present the lower ${ }_{m}$ (the higher preloading forces $F_{V}$ ). For example, in presence of unspoiled surfaces, the overall friction coefficient ${ }_{m}$ decreases from the value of 0.26 in case of forged, anodized and unlubricated specimens to the value of 0.11 in case of forged, spray-painted and unlubricated ones, so that the preloading force increases of twice with the same tightening torque $T=15 \mathrm{Nm}$. Lubrication always increases the preloading forces: in presence of forged and unspoiled surfaces, the overall friction coefficient $m$ decreases from the value of 0.11 to 0.08 in case of spray-painted specimens, and from the value of 0.26 to 0.17 in case of anodized ones. Finally, considering the effect of the number of tightening and loosening (up to six maintenance operations in a standard motorbike lifecycle) the preloading force are affected by the tightening replicas mainly in case of unlubricated and anodized surfaces so that the preloading forces progressively decrease for the same tightening torque. As a matter of fact the surfaces are subjected to wear and spoiling (Fig.7) by increasing the number of tightening, while lubrication creates a sort of protective film. In presence of spoiled surfaces, the overall friction coefficient ${ }_{m}$ increases from the value of 0.26 to 0.39 in case of forged, anodized and unlubricated surfaces, whereas from the value of 0.16 to 0.17 in case of forged, anodized and lubricated surfaces.

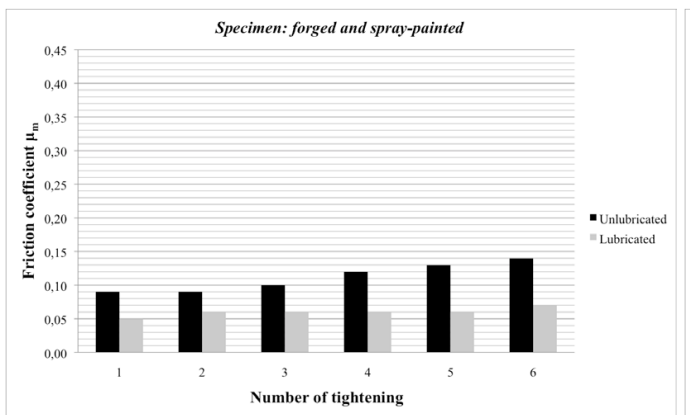

(a)

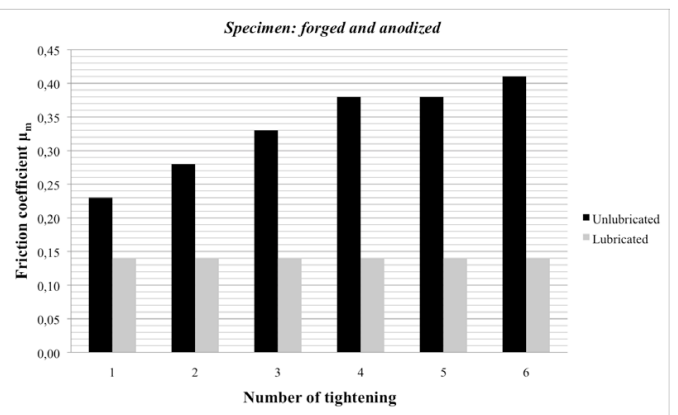

(b)

Fig.5 Bars diagrams of the friction coefficient $\mu_{m}$ values for forged specimens ((a): spray-painted (b): anodized) in case of tightening torque $T=15 \mathrm{Nm}$ (different series for lubricated and unlubricated specimens) 


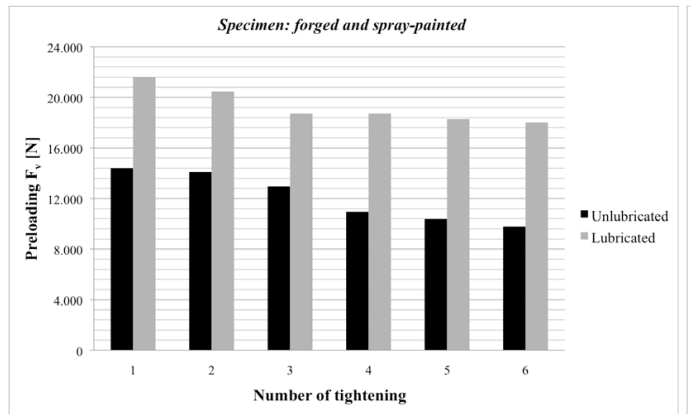

(a)

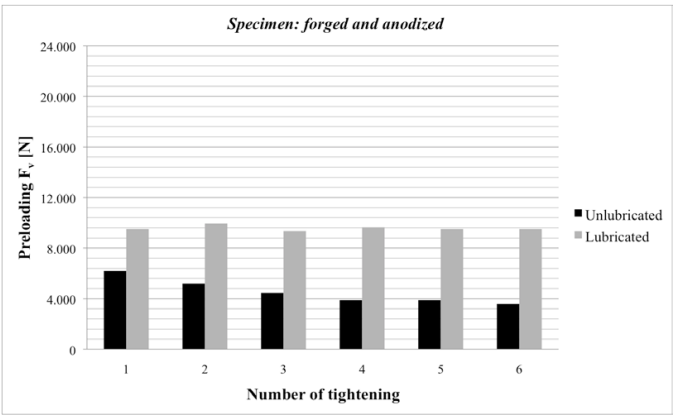

(b)

Fig.6 Bars diagrams of the preloading force $F_{V}$ values for forged specimens $((\mathbf{a})$ : spray-painted (b): anodized) in case of tightening torque $T=15 \mathrm{Nm}$ (different series for lubricated and unlubricated specimens)

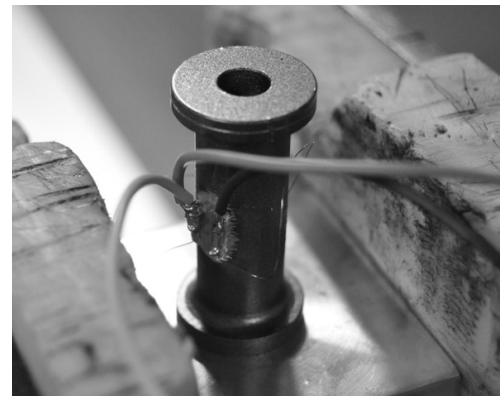

(a)

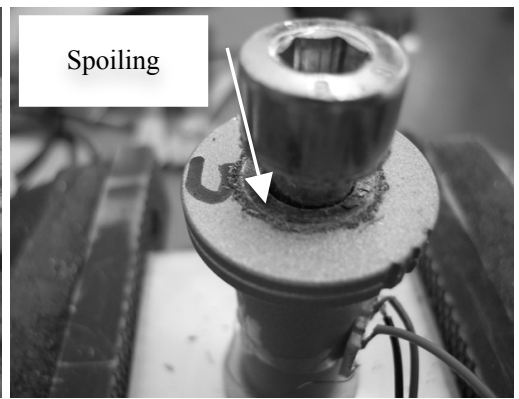

(b)

Fig.7 Spray-painted specimens (a): unspoiled surfaces with D variable equal to $0(\mathbf{b})$ : spoiled surfaces with D variable equal to 1

\section{Conclusion}

The friction coefficient and the torque coefficient have been accurately calculated for some bolted joints of components used in front motorbike suspensions. For this purpose the authors have been designed and realised some specific specimens in order to evaluate differences between cast and forged aluminium alloy, between spray painted and anodized surfaces, between lubricated and unlubricated screw and, finally, between first tightening (fresh and unspoiled surfaces) and sixth tightening (spoiled surfaces). A total of 48 tests have been executed by applying the DOE method while the results have been analysed by the ANOVA and F-Test methods: friction conditions are strongly affected by surface finishing, lubrication and number of tightening and loosening; the forming process (forged or cast aluminium alloy) seems to have no significant influence on friction conditions. The mathematical models of the overall friction and torque coefficients have been derived as functions of the significant variables highlighted by the ANOVA and F-Test. The presented models are useful to estimate precisely the actual preloading force, deriving it directly from the tightening torque. The actual torque versus preloading force relationship for aluminiumsteel bolted joints used in front motorbike suspensions can be effectively adopted for any type of maintenance operation. 


\section{References}

1. S.A. Nassar, H. El-Khiami, G.C. Barber, Q. Zou, T.S. Sun, Journal of Tribology 127, 263-272 (2005)

2. S.A. Nassar, S. Ganeshmurthy, R.M. Ranganathan, G.C. Barber, ASME Journal of Pressure Vessel Technology 129, 426-440 (2007)

3. R.C. Juvinall, K.M. Marshek, Fundamental of Machine Component Design, 3rd ed., Wiley, New York (2000)

4. S.A. Nassar, P.H. Matin, G.C. Barber, Thread Friction Torque in Bolted Joints, ASME Journal of Pressure Vessel Technology, 127, 387-393 (2004)

5. CNR-UNI10011, Costruzioni in acciaio: istruzioni per il calcolo, l'esecuzione, il collaudo e la manutenzione (1988)

6. J.H. Bickford, An Introduction to the Design and Analysis of Bolted Joints, 3rd ed., Marcel Dekker, New York (1997)

7. N. Motosh, Development of Design Charts for Bolts Preload up to the Plastic Range, ASME J. Eng. Ind., 98, 849-851 (1976).

8. VDI Handbuch Konstruktion 2230, Systematic calculation of high duty bolted joints - Joints with one cylindrical bolt (2001).

9. D. Croccolo, R. Cuppini, N. Vincenzi, Finite Elements in Analysis and Design 45, 406-414 (2009).

10. DIN946, Determination of coefficient of friction of bolt/nut assemblies under specified conditions (1991)

11. G. Niemann, H. Winter, B.R. Hohn, Maschineelemente B.d. I. Springer-Verlag, Berlin (2005)

12. D.C. Montgomery, Design and Analysis of Experiment, 5th ed. Wiley, New York (2001)

13. R. De Vor, T.H. Chang, J.W. Sutherland, Statistical Quality Design and Control, Maxwell Mcmillan International Edition, New York (1992)

14. D. Croccolo, R. Cuppini, N. Vincenzi, Strain 44, 170-179 (2008) 